\title{
Spectroscopic study of light scattering in linear alkylbenzene for liquid scintillator neutrino detectors
}

\author{
Xiang Zhou ${ }^{1, a}$, Qian Liu ${ }^{2, b}$, Junbo Han ${ }^{3}$, Zhenyu Zhang ${ }^{1}$, Xuan Zhang ${ }^{4}$, Yayun Ding ${ }^{4}$, Yangheng Zheng ${ }^{2}$, \\ Li Zhou ${ }^{4}$, Jun Cao ${ }^{4}$, Yifang Wang ${ }^{4}$ \\ ${ }^{1}$ Hubei Nuclear Solid Physics Key Laboratory, Key Laboratory of Artificial Micro- and Nano-structures of Ministry of Education and School of \\ Physics and Technology, Wuhan University, Wuhan 430072, China \\ ${ }^{2}$ School of Physics, University of Chinese Academy of Sciences, Beijing 100049, China \\ ${ }^{3}$ Wuhan National High Magnetic Field Center, Huazhong University of Science and Technology, Wuhan 430074, China \\ ${ }^{4}$ Institute of High Energy Physics, Chinese Academy of Sciences, Beijing 100049, China
}

Received: 16 April 2015 / Accepted: 6 November 2015 / Published online: 21 November 2015

(C) The Author(s) 2015. This article is published with open access at Springerlink.com

\begin{abstract}
We have set up a light scattering spectrometer to study the depolarization of light scattering in linear alkylbenzene. The scattering spectra show that the depolarized part of light scattering is due to Rayleigh scattering. The additional depolarized Rayleigh scattering can make the effective transparency of linear alkylbenzene much better than expected. Therefore, sufficient scintillation photons can transmit through large liquid scintillator detector, such as that of the JUNO experiment. Our study is crucial to achieving an unprecedented energy resolution of $3 \% / \sqrt{E(\mathrm{MeV})}$ required for the JUNO experiment to determine the neutrino mass hierarchy. The spectroscopic method can also be used to examine the depolarization of other organic solvents used in neutrino experiments.
\end{abstract}

\section{Introduction}

Organic scintillators have played a key role in the 60-yearlong research history of neutrinos [1]. They were chosen as the targets in the liquid scintillator detectors where antielectron neutrinos were first discovered by Cowan and Reines [2], in the KamLAND experiment where the large mixing angle (LMA) solution of the solar neutrino problem was confirmed [3], in the Daya Bay experiment where the neutrino oscillation mixing angle $\theta_{13}$ was precisely measured [4], and in the Borexino experiment where the spectrum of protonproton neutrinos was recently observed [5]. Because of the unexpectedly large value of $\theta_{13}$, the neutrino mass hierarchy and the leptonic $C P$-violating phase can be measured in the next generation of neutrino oscillation experiments [6].

\footnotetext{
a e-mail: xiangzhou@whu.edu.cn

b e-mail: liuqian@ucas.ac.cn
}

Recently, the Jiangmen Underground Neutrino Observatory (JUNO) experiment has been approved, aiming to measure the neutrino mass hierarchy using an organic liquid scintillator detector [7]. There will be 20 kilotons of liquid scintillator filling the huge spherical central detector of JUNO, whose diameter will be about $35 \mathrm{~m}$. A ternary hydrogenous organic liquid is chosen as the detector target in JUNO, with the solvent being linear alkylbenzene (LAB), whose formula is $\mathrm{C}_{6} \mathrm{H}_{5} \mathrm{C}_{n} \mathrm{H}_{2 n+1}(\mathrm{n}=10-13)$, and the primary and secondary solutes being 2,5-diphenyloxazole (PPO) and 1,4bis[2-methylstyryl]benzene (bis-MSB), respectively. Reactor anti-neutrinos can be detected via the inverse beta-decay reaction $\bar{v}_{e}+p \rightarrow e^{+}+n$. Each positron will rapidly annihilate with an electron and produce a pair of $\gamma$-rays. Each neutron will be finally captured by a proton and release a $\gamma$-ray. The $\gamma$-rays will product scintillation in the LAB. The solutes, PPO and bis-MSB, will transfer the wavelengths of scintillation to longer wavelength to avoid self-absorption by the LAB [8]. In the discussion of light transmission in the liquid scintillator, the reference wavelength is usually chosen as $430 \mathrm{~nm}$, around which the intensity of the scintillation spectrum is at a maximum.

The JUNO experiment will determine the neutrino mass hierarchy by measuring a very precise and high-statistics reactor anti-neutrino energy spectrum [9]. The method demands an unprecedented energy resolution of $3 \% /$ $\sqrt{E(\mathrm{MeV})}$, i.e., about 1200 photon electrons per $\mathrm{MeV}$ [7]. The large scale of the central detector will make scintillation photons transmit several tens of meters before they finally arrive at the photomultipliers and then transfer into photon electrons. The photons attenuate exponentially when they transmit in a liquid. The detection challenge requires that the solvent of the liquid scintillator, LAB, should be highly 
transparent to scintillation photons [6]. After purification, $\mathrm{LAB}$ can have a large attenuation length which is about 20 $\mathrm{m}$ at $430 \mathrm{~nm}$ [10]. Even if the scintillation photons vanish exponentially after transmitting $20 \mathrm{~m}$, the energy resolution can still hardly reach the requirement of the JUNO experiment. Attenuation contains two sub-processes, scattering and absorption [11]. Since the liquid scintillator should be dustfree, the scattering process of LAB should be Rayleigh scattering. Because Rayleigh scattering only changes the directions of photons, the scattered photons still have a chance to be detected in a $4 \pi$ detector. It has been found that Rayleigh scattering can increase the effective transparency of scintillation in large liquid scintillator detectors [12]. Simulations have shown that if the Rayleigh scattering length of LAB at $430 \mathrm{~nm}$ is $30 \mathrm{~m}$, the energy resolution of the central detector of JUNO can satisfy the requirement for measurement of the neutrino mass hierarchy [13].

Light scattering in several organic solvents, including $\mathrm{LAB}$, has been studied experimentally [14]. It has been found that light scattering in LAB is depolarized at $90^{\circ}$. It is well known that the Rayleigh scattering of light by small isotropic dielectric spheres at $90^{\circ}$ is completely polarized [15]. The Rayleigh scattering length of LAB will be about $40 \mathrm{~m}$ at 430 $\mathrm{nm}$ if only the polarized part of light scattering is considered. The depolarized part of light scattering in LAB has been treated as a result of absorption/reemission [14]. Because $430 \mathrm{~nm}$ is far from the absorption band of LAB [8], the weak absorption including the absorption/reemission of LAB at $430 \mathrm{~nm}$ is due to the optical purities [16,17]. Recently, it has been argued that the depolarized part of light scattering in LAB is also Rayleigh scattering because of molecular anisotropy [18]. Then the depolarization of light scattering in LAB is an intrinsic property of LAB. A significant difference between the depolarized Rayleigh scattering and absorption/reemission is that the wavelengths of the deflected photons will remain in the former process and change in the latter [19]. Whatever the depolarized part of light scattering is, the total scattering length of the LAB in the previous experiment [14] will shorten to about $30 \mathrm{~m}$ at $430 \mathrm{~nm}$ because the quantum efficient of photomultipliers is typically flat around $430 \mathrm{~nm}$. However, optical purities in LAB of different productions or purifications are various in types and amounts. The LAB in the JUNO experiment may not have the same absorption/reemission as the $\mathrm{LAB}$ in the previous experiment. Therefore, the total scattering length of LAB in the JUNO experiment will be guaranteed to be less than $30 \mathrm{~m}$ at $430 \mathrm{~nm}$ [18] only if the depolarized part of light scattering is Rayleigh scattering.

To distinguish whether the depolarized part of light scattering in LAB is Rayleigh scattering or absorption/reemission is crucial for the JUNO experiment to achieving the unprecedented energy resolution of $3 \% / \sqrt{E(\mathrm{MeV})}$ to measure the neutrino mass hierarchy. In this paper, we stud- ied spectroscopically the depolarization of light scattering in LAB. The experimental setup used for the scattering spectrum measurements is described in Sect. 2. Results and discussions of the scattering spectra and the depolarization of LAB are presented in Sect. 3. A summary is given in Sect. 4 .

\section{Experimental setup}

A schematic diagram of the light scattering spectrometer is shown in Fig. 1. The light source was a commercial SpectraPhysics BeamLok 2060 argon ion continuous-wave laser. The beam from the laser had a divergence of $0.45 \mathrm{mrad}$ with a beam diameter of $1.7 \mathrm{~mm}$. The light beam supplied by the laser was vertically polarized with the extinction ratio better then 100:1.

The beam first passed through a circular continuously variable reflective neutral density filter which can adjust light intensity continuously. The beam was then collimated by two reflection mirrors and two apertures. Between the mirrors and apertures, a beam splitter was used to split the beam into two parts. One part of the beam remained on the original path and the other, perpendicular to the original path, was received by a power meter to monitor the stability of the laser beam. Since the optical components would induce spurious depolarization, a Glan-Laser calcite polarizing prism was put behind the splitter to improve the extinction ratio to be better than 5000:1. After passing through two apertures the laser beam enter a cell whose light path was $50 \mathrm{~mm}$. The cell was of strain-free fused quartz of very high optical quality. The cell was mounted on an optical bench whose height could be adjusted for final alignment of the cell with respect to the incident beam. This alignment was not changed during the experiments. The cell, which could be emptied or filled with $\mathrm{LAB}$, was held firmly in position during measurements by a removable spring yoke. The attenuation length of the batch of LAB sample was about $20 \mathrm{~m}$ [10].

Following the cell, two apertures, with holes of diameter $1 \mathrm{~mm}$ normal to the laser beam, served to limit the field of view of the spectroscope and prevent stray light from enter-

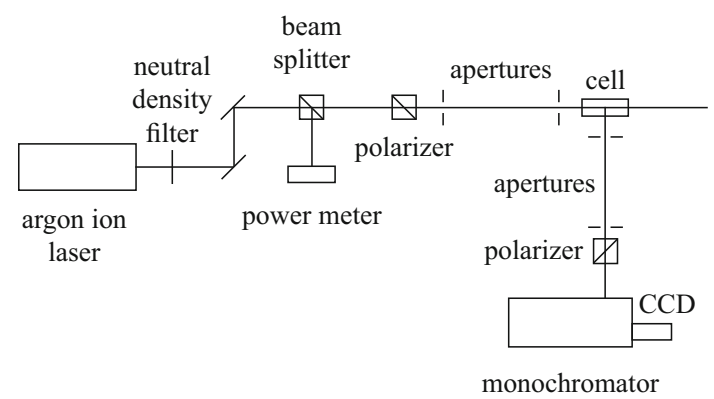

Fig. 1 A schematic diagram of the light scattering spectrometer 
ing the spectroscope. The first aperture was $65 \mathrm{~mm}$ behind the cell. The second aperture was $790 \mathrm{~mm}$ after the first aperture. A Glan-Thompson calcite polarizer was mounted on a motorized precision rotation mount (Thorlabs PRM1/MZ8) $115 \mathrm{~mm}$ behind the second aperture. The scattered photons entered the slit of a Shamrock SR-500i grating monochromator which was $53 \mathrm{~mm}$ behind the calcite polarizer. The light was finally collected by an Andor Newton EMCCD DU971P CCD detector. The temperature of the CCD detector was cooled to $-75{ }^{\circ} \mathrm{C}$ to decrease noise. The experiment was held in a clean room with room temperature controlled at $21 \pm 1^{\circ} \mathrm{C}$. The scattered photons were collected horizontally and vertically separately by the Glan-Thompson calcite polarizer. The horizontal and vertical scattering spectra were given by the spectroscope with the CCD detector.

\section{Results and discussion}

Firstly, the output wavelength of the argon ion laser was set to $488 \mathrm{~nm}$ and the cell was empty. The slit width was set to 0.2 $\mathrm{mm}$ and then the spectroscopic resolution would be about 1 $\mathrm{nm}$. In order to make the laser operate stably, the output power was set to the optimized one which was approximately 300 $\mathrm{mW}$ at $488 \mathrm{~nm}$. The output beam power was so high that for safety a reflective neutral filter was used to reduce the input laser intensity to the organic sample. The filter reflected about $99.6 \%$ laser light. Although a light shield was constructed from the cell to the CCD detector, there were still peaks around $488 \mathrm{~nm}$ in the spectra caused by stray light. The vertical and horizontal scattering spectra for the empty cell with an integration time of $5 \mathrm{~s}$ are shown in Fig. 2. The maximum intensities of both spectra were near to each other because the stray light had been reflected so many times that it would be almost unpolarized. The flat intensities of scattering spectra away from $488 \mathrm{~nm}$ were caused by the CCD detector noise.

The cell was then filled with LAB without any movement. The vertical and horizontal scattering spectra for LAB with an integration time of $5 \mathrm{~s}$ are shown in Fig. 3. Because of light scattering from $\mathrm{LAB}$, the intensities of peaks were larger than those for the empty cell. All the wavelengths of peaks in the scattering spectra in Figs. 2 and 3 were exactly the same. Thus it can be concluded that the scattered photons from LAB are caused by Rayleigh scattering.

In the light of the above spectroscopic study, the peak background in the spectra caused by stray light has to be suppressed for quantitative analysis of the depolarization of Rayleigh scattering in LAB. The output wavelength of the argon ion laser was then set to $457.9 \mathrm{~nm}$ so that the output power could be lowered to approximately $50 \mathrm{~mW}$. Stray light in the lab was successfully suppressed. However, very few scattered photons could be collected by the spec-

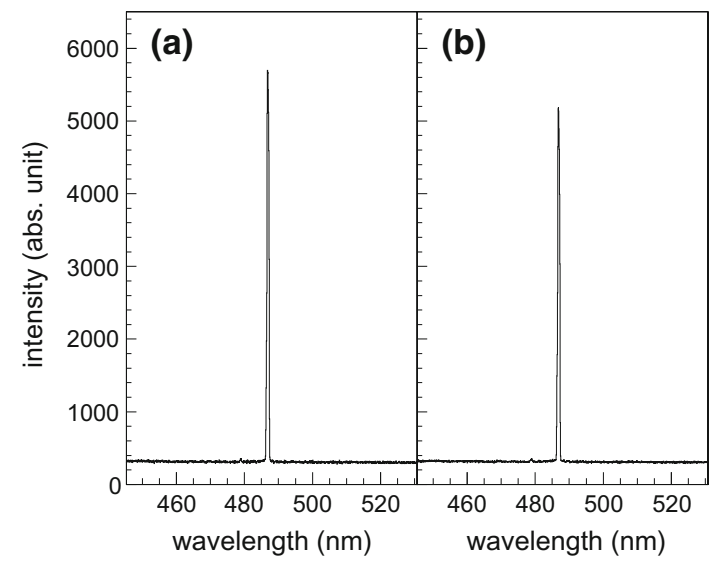

Fig. 2 a The vertical scattering spectrum and $\mathbf{b}$ the horizontal scattering spectrum of the empty cell for $488 \mathrm{~nm}$ incident laser beam. The slit width was $0.2 \mathrm{~mm}$ and the integration time of the CCD was $5 \mathrm{~s}$

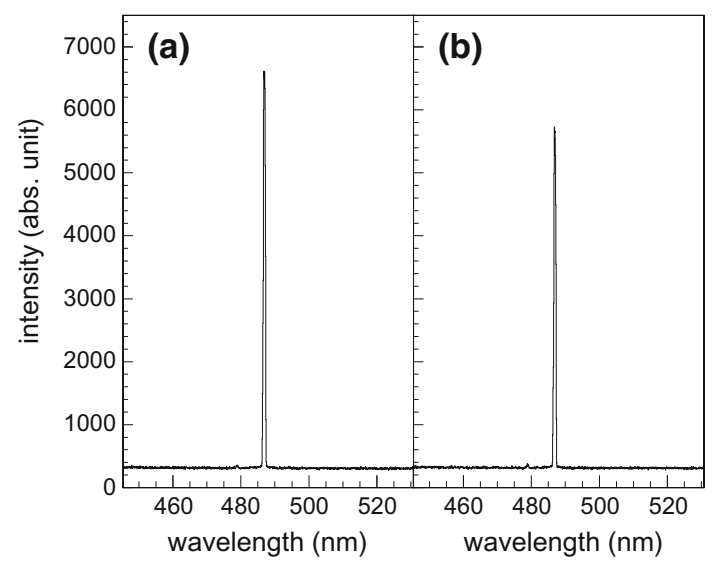

Fig. 3 a The vertical scattering spectrum and $\mathbf{b}$ the horizontal scattering spectrum of LAB for $488 \mathrm{~nm}$ incident laser beam. The slit width was $0.2 \mathrm{~mm}$ and the integration time of the CCD was $5 \mathrm{~s}$

troscope because of the $0.2 \mathrm{~mm}$ narrow slit. The slit width was then widened to $1 \mathrm{~mm}$ and the integration time of the $\mathrm{CCD}$ increased to $20 \mathrm{~s}$. The widened slit lowered the spectroscopic resolution to about $5 \mathrm{~nm}$. The fluorescence spectra of a mixture of LAB and bis-MSB have shown that the spectroscopic properties of the Rayleigh scattering of LAB and the absorption/reemission of bis-MSB are totally different [19]. Thus if there was absorption/reemission, it can still be distinguished from Rayleigh scattering in the scattering spectra. The vertical and horizontal scattering spectra of the empty cell are shown in Fig. 4. The horizontal scattering spectrum was almost flat, while there was only a very small peak around $457.9 \mathrm{~nm}$ in the vertical scattering spectrum which might be caused by light scattering from air or cell.

The vertical and horizontal scattering spectra for LAB are shown in Fig. 5. There are obvious peaks around $457.9 \mathrm{~nm}$ in the vertical and horizontal scattering spectra. The depolarization of Rayleigh scattering in LAB can be characterized by 


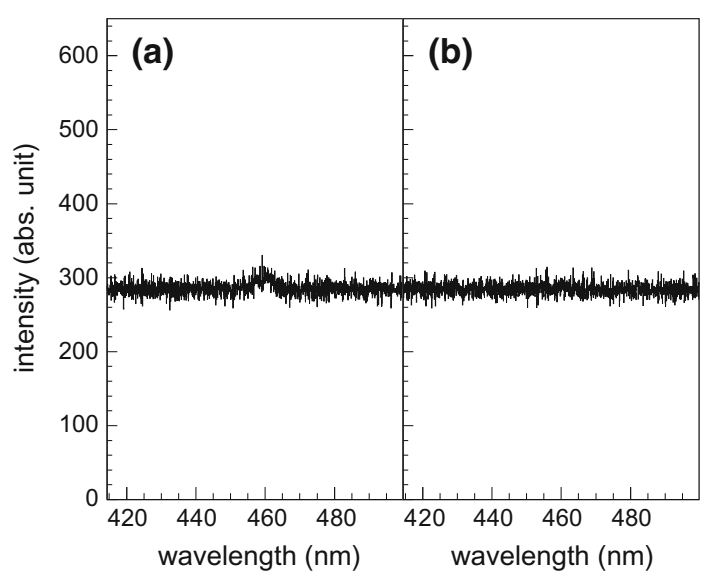

Fig. 4 a The vertical scattering spectrum and $\mathbf{b}$ the horizontal scattering spectrum of the empty cell for $457.9 \mathrm{~nm}$ incident laser beam. The slit width was $1 \mathrm{~mm}$ and the integration time of the CCD was $20 \mathrm{~s}$

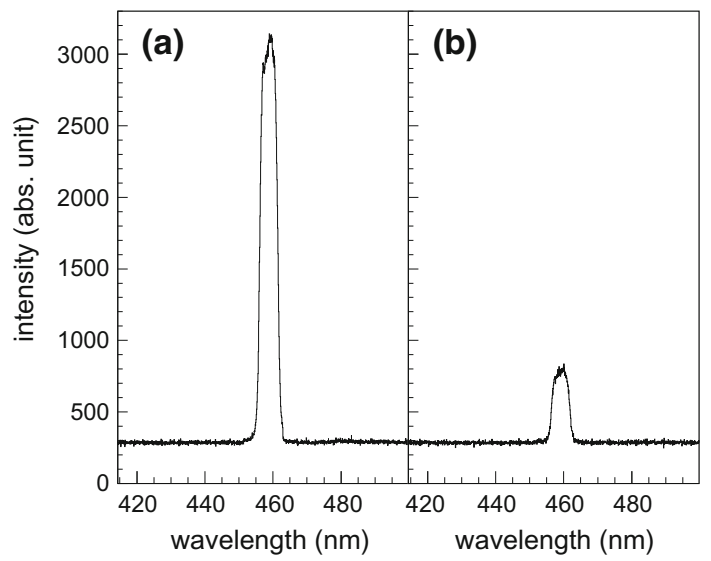

Fig. 5 a The vertical scattering spectrum and $\mathbf{b}$ the horizontal scattering spectrum of LAB for $457.9 \mathrm{~nm}$ incident laser beam. The slit width was $1 \mathrm{~mm}$ and the integration time of the CCD was $20 \mathrm{~s}$

the depolarization ratio [20]. The directly measured quantity from the scattering spectra is

$\rho_{v}=\frac{H_{v}}{V_{v}}$,

where $H_{v}$ and $V_{v}$ are the horizontal and vertical intensities of the scattered photons from a vertically polarized incident beam. The depolarization ratio $\rho_{u}$ of an unpolarized incident beam is defined as

$\rho_{u}=\frac{H_{v}+H_{h}}{V_{h}+V_{v}}$,

where $H_{h}$ and $V_{h}$ are the horizontal and vertical intensities of the scattered photons from a horizontal polarized incident beam. When the scattering angle is $90^{\circ}, H_{v}=H_{h}=V_{h}$ and then the depolarization ratio $\rho_{u}$ can be obtain by
Table 1 Depolarization ratio of LAB

\begin{tabular}{llll}
\hline$\rho_{v}(457.9 \mathrm{~nm})$ & $\begin{array}{l}\rho_{u}(457.9 \mathrm{~nm}) \\
\text { Present work }\end{array}$ & $\begin{array}{l}\rho_{u}(430 \mathrm{~nm}) \\
\text { Pef. [18] }\end{array}$ & $\begin{array}{l}\rho_{u}(405 \mathrm{~nm}) \\
\text { Ref. [22] }\end{array}$ \\
\hline $0.18 \pm 0.01$ & $0.30 \pm 0.02$ & $0.31 \pm 0.04$ & $0.31 \pm 0.02$ \\
\hline
\end{tabular}

$\rho_{u}=\frac{2 \rho_{v}}{1+\rho_{v}}$,

which is the specific result for Krishnan's relations at the $90^{\circ}$ scattering angle [21].

The scattering intensities $H_{v}$ and $V_{v}$ can be obtained from the scattering spectra by subtracting the noise background. The results derived from Eqs. (1) and (2) are listed in Table 1. The scattering spectra of LAB were measured three times. The statistical uncertainty of the depolarization ratio is about $3 \%$. The bidirectional repeatability of the polarization angle controlled by a precision rotation mount is $\pm 0.1^{\circ}$ and it causes an error of $1 \%$. The stability of the laser beam was monitored by a power meter during the measurement and the instability of the laser beam causes an error of $1 \%$. Therefore, the depolarization ratio of LAB is measured to be 0.30 \pm 0.02 at $457.9 \mathrm{~nm}$.

Recently the depolarization ratio of LAB at $405 \mathrm{~nm}$ has also been measured independently giving a result of $0.31 \pm$ 0.02 [22]. From Table 1, the depolarization ratios of 405 , 430 and $457.9 \mathrm{~nm}$ for LAB are the same within $1 \sigma$. It is well known that the depolarization ratio is a weak function of wavelength over the visible range [20,23]. Therefore, we can conclude that the depolarized part of light scattering in LAB is Rayleigh scattering even if we can not directly study the depolarization of light scattering of LAB at $430 \mathrm{~nm}$, the main region of interest, due to the limitation of light source. Then the total light scattering length of Rayleigh scattering in LAB should be less than $30 \mathrm{~m}$ at $430 \mathrm{~nm}$ [18].

Experimentally, even gases can have very small depolarization ratios due to molecular anisotropy [24]. The light scattered by liquids is generally depolarized $[25,26]$. Polarized Rayleigh scattering has been used to approximately describe light scattering in water Cherenkov detectors $[11,27]$ and rare-gas liquid detectors [28] because the depolarization ratio of water and rare gas liquids are small. However, the depolarization of Rayleigh scattering in LAB is too large to be ignored. The typical fluorescence time of absorption/reemission for liquid scintillators is several nano-seconds to which large detectors are sensitive $[29,30]$. To treat the depolarized part of Rayleigh scattering as absorption/reemission would increase the time response in simulations of large scintillator detectors and might affect the spatial resolutions and the detection efficiencies $[31,32]$. Aside from the JUNO experiment [9], LAB has been used in the Daya Bay experiment [33], RENO experiment [34] and the upcoming SNO+ exper- 
iment $[35,36]$. It would also be used in the planned LENA [37] and Hanohano observatories [38].

The previous scattering experiment has also shown that the light scatterings in dodecane, pseudocumene (PC) and phenylxylylethane (PXE) are also depolarized [14]. PC is currently used as the solvent in the Borexino experiment $[32,39]$ and PXE has been investigated as a back-up solution for the Borexino experiment [40]. The solvent used in KamLAND experiment consists of $20 \% \mathrm{PC}$ and $80 \%$ normal dodecane (ND) [12]. The diameters of the liquids scintillator detectors in Borexino and KamLAND are 8.5 and 13 m, respectively. If the depolarized parts are also Rayleigh scattering, the scattering lengths of PXE and PC will shorten to about $10 \mathrm{~m}$ and the scattering length of the mixture of PC and ND will be also shorter than expected. The spectroscopic method can be used for the Borexino and KamLAND experiments to investigate whether the depolarization of light scattering in PXE, PC and ND are due to Rayleigh scattering or absorption/reemission.

\section{Summary}

A light scattering spectrometer has been set up to investigate the depolarization of light scattering in LAB. It has been shown that the depolarized part of light scattering in LAB is due to Rayleigh scattering. The additional depolarized Rayleigh scattering can increase the effective transparency of LAB. Our study will help the JUNO experiment to achieve the unprecedented energy resolution of $3 \% / \sqrt{E(\mathrm{MeV})}$ to determine the neutrino mass hierarchy measurement. Our result is also useful to other neutrino experiments which use $\mathrm{LAB}$ as the solvent for their liquid scintillators detectors. The spectroscopic method can be also used to test the depolarization of PXE, PC and ND for the Borexino and KamLAND experiments.

Acknowledgments This work has been supported by the Major Program of the National Natural Science Foundation of China (Grant No. 11390381), the Strategic Priority Research Program of the Chinese Academy of Sciences (Grant No. XDA10010500), the 985 project of Wuhan University (Grant No. 202273344).

Open Access This article is distributed under the terms of the Creative Commons Attribution 4.0 International License (http://creativecomm ons.org/licenses/by/4.0/), which permits unrestricted use, distribution, and reproduction in any medium, provided you give appropriate credit to the original author(s) and the source, provide a link to the Creative Commons license, and indicate if changes were made. Funded by SCOAP ${ }^{3}$.

\section{References}

1. K.K. Joo, J. Korean Phys. Soc. 63, 1489 (2013)
2. C.L. Cowan Jr, F. Reines, F.B. Harrison, H.W. Kruse, A.D. McGuire, Science 124, 103 (1956)

3. K. Eguchi et al. (KamLAND Collaboration), Phys. Rev. Lett. 90, 021802 (2003)

4. F.P. An et al. (Daya Bay Collaboration), Phys. Rev. Lett. 108, 171803 (2012)

5. G. Bellini et al. (Borexino Collaboration), Nature 512, 383 (2014)

6. R.N. Cahn, D.A. Dwyer, S.J. Freedman, W.C. Haxton, R.W. Kadel, Yu.G. Kolomensky, K.B. Luk, P. McDonald, G.D. Orebi Gann, A.W.P. Poon, arXiv:1307.5487v2 (2014)

7. Y.-F. Li, J. Cao, Y. Wang, L. Zhan, Phys. Rev. D 88, 013008 (2013)

8. H.L. Xiao, X.B. Li, D. Zheng, J. Cao, L.J. Wen, N.Y. Wang, Chin. Phys. C 34, 1724 (2010)

9. Y.F. Li, Int. J. Mod. Phys. Conf. Ser. 31, 1460300 (2014)

10. L. Gao, B.X. Yu, Y.Y. Ding, L. Zhou, L.J. Wen, Y.G. Xie, Z.G. Wang, X. Cai, X.L. Sun, J. Fang, Z. Xue, A.W. Zhang, Q.W. Lü, L.J. Sun, Y.S. Ge, Y.B. Liu, S.L. Niu, T. Hu, J. Cao, J.G. Lü, Chin. Phys. C 37, 076001 (2013)

11. S. Agostinelli et al. (GEANT4 Collaboration), Nucl. Instrum. Methods Phys. Res. A 506, 250 (2003)

12. F. Suekane, T. Iwamoto, H. Ogawa, O. Tajima, H. Watanabe, arXiv:physics/0404071v2 (2004)

13. Y.F. Wang, Introduction to the JUNO experiment. The 2014 Shanghai Particle Physics and Cosmology Symposium (SPCS2014), Shanghai, May 29, 2014

14. M. Wurm, F. Von Feilitzsch, M. Göger-Neff, M. Hofmann, T. Lachenmaier, T. Lewke, T.M. Undagoitia, Q. Meindl, R. Möllenberg, L. Oberauer, W. Potzel, M. Tippmann, S. Todor, C. Traunsteiner, J. Winter, Rev. Sci. Instrum. 81, 053301 (2010)

15. L. Rayleigh, Philos. Mag. 47, 375 (1899)

16. P. Huang, P. Li, Z. Fu, C. He, Y. Ding, J. Li, M. Qi, JINST 5, P08007 (2010)

17. P.W. Huang, H.Y. Cao, M. Qi, P.Y. Li, Z.W. Fu, C.C. He, J.X. Li, Y.Y. Ding, Theor. Chem. Acc. 129, 229 (2011)

18. X. Zhou, Q. Liu, M. Wurm, Q. Zhang, Y. Ding, Z. Zhang, Y. Zheng, L. Zhou, J. Cao, Y. Wang, Rev. Sci. Instrum. 86, 073310 (2015)

19. X.F. Ding, L.J. Wen, X. Zhou, Y.Y. Ding, X.C. Ye, L. Zhou, M.C. Liu, H. Cai, J. Cao, Chin. Phys. C 39, 126001 (2015)

20. M. Kerker, The Scattering of Light and Other Electromagnetic Radiation (Academic Press, New York, 1969)

21. R.S. Krishnan, Proc. Indian Acad. Sci. Sect. A 10, 395 (1939)

22. Q. Liu, X. Zhou, W. Huang, Y. Zhang, W. Wu, W. Luo, M. Yu, Y. Zheng, L. Zhou, J. Cao, Y. Fang, Nucl. Instrum. Methods Phys. Res. A 795, 284 (2015)

23. R.S. Farinato, R.L. Rowell, J. Chem. Phys. 65, 593 (1976)

24. R.C. Watson, R.L. Rowell, J. Chem. Phys. 61, 2666 (1974)

25. D.J. Coumou, E.L. Mackor, J. Hijmans, Trans. Faraday Soc. 60, 1539 (1964)

26. H. Wahid, J. Opt. (Paris) 26, 109 (1995)

27. M. Shiozawa, Search for Proton Decay via $p \rightarrow e^{+} \pi^{0}$ in a Large Water Cherenkov Detector. Ph.D. thesis, University of Tokyo, Japan, 1999

28. G.M. Seidel, R.E. Lanou, W. Yao, Nucl. Instrum. Methods Phys. Res. A 489, 189 (2002)

29. T.M. Undagoitia, F. Feilitzsch, L. Oberauer, W. Potzel, A. Ulrich, J. Winter, M. Wurm, Rev. Sci. Instrum. 80, 043301 (2009)

30. X.B. Li, H.L. Xiao, J. Cao, J. Li, X.C. Ruan, Y.K. Heng, Chin. Phys. C 35, 1026 (2011)

31. F. Elisei, F. Gatti, A. Goretti, T. Hagner, F. Masetti, U. Mazzucato, G. Ranucci, S. Schoenert, G. Testera, P. Ullucci, S. Vitale, Nucl. Instrum. Methods Phys. Res. A 400, 53 (1997)

32. G. Alimonti et al. (Borexino Collaboration), Nucl. Instrum. Methods Phys. Res. A 440, 360 (2000)

33. F.P. An et al. (Daya Bay Collaboration), Nucl. Instrum. Methods Phys. Res. Sect. A. 685, 78 (2012) 
34. J.K. Ahn et al. (RENO Collaboration), Phys. Rev. Lett. 108, 191802 (2012)

35. M.C. Chen, Nucl. Phys. B (Proc. Suppl.) 145, 65 (2004)

36. M.C. Chen, AIP Conf. Proc. 944, 25 (2007)

37. M. Wurm et al. (LENA Collaboration), Astropart. Phys. 35, 685 (2012)
38. J.G. Learned, S.T. Dye, S. Pakvasa, arXiv:0810.4975 (2008)

39. G. Alimonti et al. (Borexino Collaboration), Nucl. Instrum. Methods Phys. Res., Sect. A. 600, 568 (2009)

40. H.O. Back et al. (Borexino Collaboration), Nucl. Instrum. Methods Phys. Res. Sect. A. 585, 48 (2008) 